\title{
A NEW PCR-RFLP FOR SPECIES-SPECIFIC DIAGNOSIS OF SOUTH AMERICAN ANIMAL TRYPANOSOMIASIS
}

\author{
${ }^{1}$ Liliana Torcoroma García, ${ }^{2}$ Yezid A. Ardila, \\ ${ }^{1}$ Deilis Rincón, ${ }^{1}$ Camila Durán and ${ }^{1}$ Jhancy R. Aguilar \\ ${ }^{1}$ Program of Bacteriology and Clinical Laboratory, \\ ${ }^{2}$ Program of Veterinary Medicine, \\ Universidad de Santander, Bucaramanga, Colombia
}

Received 2014-03-07; Revised 2014-06-04; Accepted 2014-06-05

\begin{abstract}
The diagnosis of animal trypanosomiasis in field samples is currently based on clinical suspicion and less frequently on traditional parasitological methods characterized by their very low sensitivity, especially in the subacute and chronic phase of the infection. Molecular tools such as Polymerase Chain Reaction have been shown to reach the highest sensitivity percentages, but without good resolution for all of the South American circulating species (T. vivax, T. evansi and T. theileri) or with specificity for only one of these species. In this study, a new PCR-RFLP for species-specific diagnosis of Trypanosomiasis is presented. This method displayed good resolution, sensitivity and specificity for differentiation of the three aforementioned species identified from naturally infected Colombian bovines and water buffaloes. In addition, epidemiological and clinical data were also collected and analyzed from the sampled animals and significant associations were identified.
\end{abstract}

Keywords: Animal Trypanosomiasis, PCR-RFLP, Trypanosoma Vivax, Trypanosoma Evansi, Trypanosoma Theileri

\section{INTRODUCTION}

Animal Trypanosomiasis is a widely distributed disease that affects multiple species of mammals, including bovines and buffaloes. In Africa, this infection is caused by Trypanosoma congolense, T. vivax, T. evansi, T. simiae and T. brucei brucei. In South America, the absence of the cyclic vector (tsetse fly) has limited the number of species involved in the domestic ruminant sickness, with only $T$. vivax and $T$. evansi being identified (Desquesnes et al., 2013). T. theileri infection has also been reported in Africa and South America, however its pathogenicity in domestic large ruminants is still under study (Lee et al., 2013).

In South America, Colombia ranks third in livestock production, this economic activity being responsible for almost 5\% of the National Gross Domestic Product. However, in this country, the economic losses due to
Animal Trypanosomiasis were estimated at US\$6 million as a consequence of lost production, treatment, prophylaxes and veterinary attention costs. This infection is endemic in the Atlantic Coast, Orinoquia, Cauca and Inter-Andin Valley or Magdalena Medio Regions (Wells et al., 1982; Otte et al., 1994; Cassalett et al., 2011). All of these areas have tropical climates characterized by high temperatures accompanied with humidity and wet weather, year round.

Despite its prevalence in Colombia and other South American nations, the diagnosis of Animal Trypanosomiasis in field samples is currently based on clinical suspicion. The principal disadvantage of this proach is the nonspecific symptoms of Trypanosomiasis, which are similar to other relevant hemoparasitosis present in the same regions, such as Anaplasmosis and Babesiosis (De Souza Pimentel et al., 2012; Desquesnes et al., 2013). Furthermore, the use of Corresponding Author:Liliana Torcoroma García, Program of Bacteriology and Clinical Laboratory,

Universidad de Santander, Bucaramanga, Colombia 
traditional parasitological methods, such as the Buffy Coat Technique-BCT (Murray et al., 1977), the MicroHematocrit Centrifugation Technique or MHCT (Woo, 1970) and the Peripheral Blood Smear (PBS) stained with Giemsa, are very limited because of their very low sensitivity, especially in the sub-acute and chronic phases of the infection, where the parasites are frequently hosted within tissues.

In the last two decades, molecular tools such as Polymerase Chain Reaction (PCR) have been shown to achieve higher rates of sensitivity (close to 100\%) for Trypanosome DNA amplification (Geysen et al., 2003; De Souza Pimentel et al., 2012). However, most of the available methods are specific to the determination of species involved in African Animal Trypanosomiasis, with poor resolution for the circulating species in the South American variant (Delespaux et al., 2003; Geysen et al., 2003). In other cases, the molecular tools described are specific to only one of the species (Mekata et al., 2009; De Souza Pimentel et al., 2012). In order to contribute to the generation of new tools for species-specific diagnosis of Trypanosomiasis from field samples of bovines and buffaloes, in this study we design a new PCR-RFLP adjusted to etiological species described in South America (T. vivax, T. evansi and $T$. theileri). The efficiency of this method was verified on 206 samples collected from bovines and buffaloes naturally infected in the Colombian Magdalena Medio Region. The results of this study were that the implemented assay presented good resolution, sensitivity and specificity for differentiation of the three species mentioned. The etiology of the infection was contrasted with the epidemiological and clinical data collected from the sampled animals.

\section{MATERIALS AND METHODS}

\subsection{Collection of Blood Samples from Domestic Ruminants}

Adult cattle and water buffalo (2 years old or older) of both sexes were bled from eleven (11) farms in four Colombian Magdalena Medio departments (6 farms in South Santander, 2 in North Santander, 2 in Cesar and 1 in Antioquia Department). This region is characterized by low altitude ( $<200 \mathrm{MASL})$, large flat areas with high humidity (rainfall mean of $2653 \mathrm{~mm} /$ year and $75 \%$ relative humidity) year-round. Their geographic location is shown in Fig. 1. The farms were chosen by convenience and the animals on each farm were randomly selected. A total of 206 EDTA whole-blood samples (86 from bovines and 120 from water buffaloes) were collected from the jugular vein during the period between November 2012 and May 2013. Relevant data from semiology, epidemiological and environmental aspects, were also collected. The health of the cattle was monitored through veterinary attention and a comparison of the hematological values of each animal was made using the reference range described by Janis, 1993. Finally, all samples were taken between 07:00-11:00 h, transported on ice, protected from light and processed within $6 \mathrm{~h}$ of collection.

\subsection{Hematological Analysis}

EDTA whole blood samples were used for hematological analysis. The Red Blood Cells (RBC) and total White Blood Cells (WBC) were counted in Neubauer's hemocytometer. The Packed Cell Volume (PCV) was determined using the micro-hematocrit technique. Blood cell morphology, differential leukocyte count and platelet count were performed on PBS stained with May-Gruwald-Giemsa and evaluated as previously described by (Coles, 1986). Direct hemoparasitological analysis under light microscope (X200) was made by MHCT (Woo, 1970), BCT (Murray et al., 1977; Holland et al., 2001) and PBS stained with Hemacolor (Merck), in accordance with the manufacturer's instructions.

\subsection{DNA Extraction}

EDTA whole-blood samples were used for DNA extraction using a QIAamp DNA Blood Mini Kit (Qiagen) in accordance with the manufacturer's instructions. The DNA was quantified using a NanoDrop ND-1000 UV-Vis Spectrophotometer (Thermo Scientific) and then stored at $-20^{\circ} \mathrm{C}$ until molecular analysis. All samples were processed for DNA extraction within six month of collection.

\subsection{PCR-RFLP Assays}

For the optimal PCR primers design and restriction enzymes search, we used the $18 \mathrm{~S}$ rRNA sequences stored in the GenBank ${ }^{\mathrm{TM}}$ (http://www.ncbi.nlm.nih.gov/genbank) for relevant South American isolated Trypanosomas: T. evansi (AF165117), T. vivax (AY362546; AY363164, AY363165; HM209400; DQ317416; DQ317415; DQ317413) and T. theileri (AY773690-AY773697; AY773674-AY773683). For the PCR assay, we used the sense primer 18SsuF1 (5'CTGCAAACGATGACACCCATGAATTG-3’)

coupled with an anti-sense primer $18 \mathrm{STnR} 3$ proposed by (Geysen et al., 2003). 


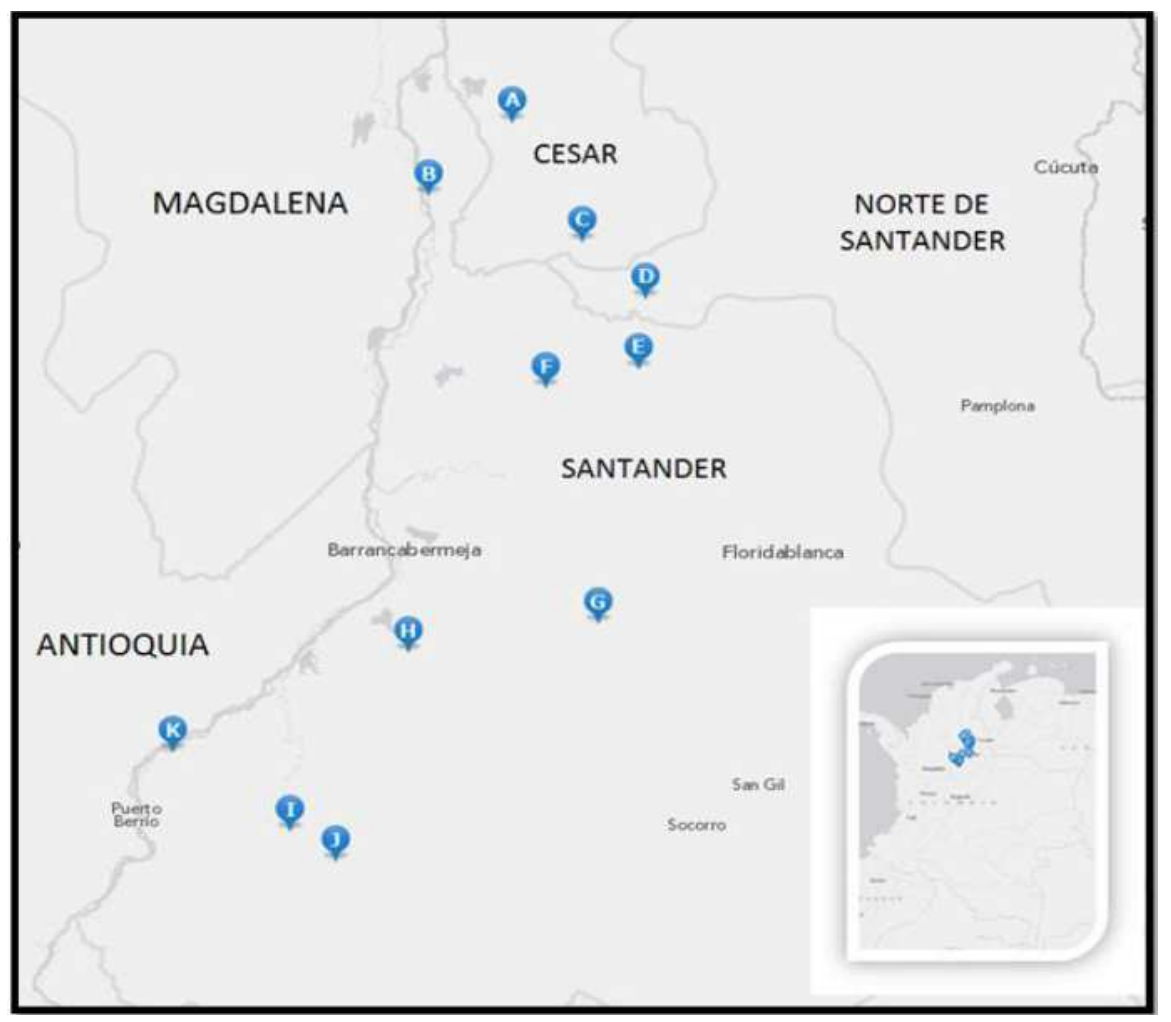

Fig. 1. Geographic location of sampled farms in Colombian Magdalena Medio Region

In the semi-nested PCR, the $18 \mathrm{SsuF} 1$ was used as sense with the anti-sense primer 18SsuR2 (5'CTTGTTCTCACTGACATTGTAGTGCGCG-3’). For the PCR and semi-nested PCR reactions, we used an amplification mixture containing: $4 \mu \mathrm{L}$ of template $(50-$ 200 ng DNA), $0.4 \mathrm{mM}$ of each primer, $0.5 \mathrm{U}$ of GoTaq Flexi DNA Polymerase (Promega) and $1.5 \mathrm{mM} \mathrm{MgCl} 2$, in a final volume of $25 \mu \mathrm{L}$. Cycling reactions were performed under the following conditions: One cycle of $2 \mathrm{~min}$ at $94^{\circ} \mathrm{C}$, followed by 30 cycles of $45 \mathrm{~s}$ at $94^{\circ} \mathrm{C}, 60$ $\mathrm{s}$ at $55^{\circ} \mathrm{C}$ and $60 \mathrm{~s}$ at $72^{\circ} \mathrm{C}$. Finally, one postamplification step of $7 \mathrm{~min}$ at $72^{\circ} \mathrm{C}$ was carried out. As positive control for molecular assays, DNA isolated from ovines and bovines experimentally infected with $T$. evansi and $T$. vivax were used. All the PCR amplicons were further analyzed by RFLP reaction. For that process, $10 \mathrm{U} / \mu \mathrm{g}$ of $B g l \mathrm{I}$ in Buffer 3 from New England Biolabs (NEB) were used on 0.3-0.6 $\mu \mathrm{g}$ of the semi-nested PCR DNA. After incubation $\left(3 \mathrm{~h}\right.$ at $37^{\circ} \mathrm{C}$ ), the total reaction volume $(15 \mu \mathrm{L})$ was applied to $2 \%$ agarose gels and revealed by SYBR Gold stained (Molecular Probe, Invitrogen Life Technologies) with $1 \mathrm{~Kb}$ and $100 \mathrm{pb}$ DNA Ladder (MBI Fermentas, Lithuania) as a molecular weight marker. The amplicons from eight PCR reactions with different RFLP profiles were ligated to the cloning vector pCR 2.1 TOPO (Invitrogen Life Technologies). The sequence of the insert was confirmed by DNA sequencing and a search by homology was then implemented using the BLASTn algorithm (http://www.ncbi.nlm.nih.gov/BLAST) (Altschul et al., 1990).

\subsection{Statistical Analysis}

The data was digitalized in an EpiData 3.1 database (EpiData Software) and analyzed by Stata/IC 12.1 (StataCorp). We performed a descriptive analysis using frequency measures of central tendency and dispersion for continuous variables and proportions for categorical variables. The normality of the continuous variables was evaluated through a Shapiro-Wilk test and a chi-square test was used to determine statistically significant differences between infected and healthy animals for each one of the independent variables. The level of significance was determined at $\mathrm{p}<0.05$. The Odds Ratio (OR) was calculated as a measure of association by a multivariate logistic regression. The agreement between microscopy and PCR was made by Cohen's Kappa with 
a confidence interval of $95 \%$. Estimating sensitivity and specific positive and negative predicted values of the MHCT assay in the identification of the Trypanosoma species was calculated according to Bayes theorem.

\subsection{Ethical Considerations}

In this study, the names of farms and their owners were not recorded during the process to maintain data confidentiality and to adhere to the International Ethical Guidelines for Biomedical Research involving animal subjects. The farms were designated with a letter from $\mathrm{A}$ to $\mathrm{K}$ in the same order as the sampling. All sampling proceedings were carried out following the guidelines of the Ethical Committee of the Universidad de Santander. All farm owners signed consent to include their animals in this study.

\section{RESULTS}

\subsection{Diagnosis of Trypanosome Infection by MHCT and PCR}

The PCR assays carried out on the positive controls and samples resulted in bands of approx. 708 to $763 \mathrm{bp}$ for the PCR and 614 to $651 \mathrm{bp}$ for the semi-nested reaction. The trypanosome infection was detected by MHCT in 5\% (6/120) and 5.8\% (5/86) of the bovine and buffalo samples, respectively; while $45 \%$ of the bovine (54/120) and buffalo (39/86) samples were positive by PCR (Table 1). The resulting values obtained from the two tests evidenced a statistically significant difference, both in the general evaluation $(\mathrm{p}<0.001)$ and the single analysis of the samples $(\mathrm{P}=0.005$ for bovines and $\mathrm{P}=$ 0.01 for buffalo). The agreement between the two diagnostic assays was also evaluated, obtaining a
Cohen's Kappa value of 0.13 (IC95\% 0.06-0.20). In this study, for the diagnostic accuracy evaluation of the MHCT test, PCR was taken as the reference test $(100 \%$ sensitivity) due to its higher detection capability. As a result, the MHCT sensitivity was $11.8 \%$ (IC95 6.1$20.2 \%$ ), with $100 \%$ specificity (IC95 96.8-100\%), a positive predictive value of $100 \%$ (IC95 71.5-100\%) and a negative predictive value of 57.9\% (IC95 50.7-65.0\%).

\subsection{Determination of Trypanosoma Species by RFLP}

All of the positive PCR samples were analyzed by RFLP for differentiation of Trypanosome species. In this study, three different species of Trypanosome were detected. In bovines, 28/120 (23\%) of the samples were infected by T. vivax, 13/120 (11\%) by T. evansi and 13/120 (11\%) by $T$. theileri. Of the buffalo samples, T. vivax, $T$. evansi and $T$. theileri were found in 24/86 (28\%), 5/86 (6\%) and 10/86 (12\%), respectively (Table 2 and Fig. 2).

\subsection{Clinical and Hematological Features of Trypanosomiasis in Bovines and Buffaloes}

Animals that tested positive for pathogenic species of Trypanosome were classified as being in the acute or chronic phases of infection in accordance with the MHCT positivity and the clinical findings (physical examination and hematological parameters). Animals with detectable parasitemia by MHCT were considered to be acute trypanosomiasis cases $(6 / 54,11 \%)$. All of these cases were also positive for the molecular test. Otherwise, animals with only positivity by PCR but with no parasitemia, were considered to be in the chronic phase of infection (48/54, 89\%).

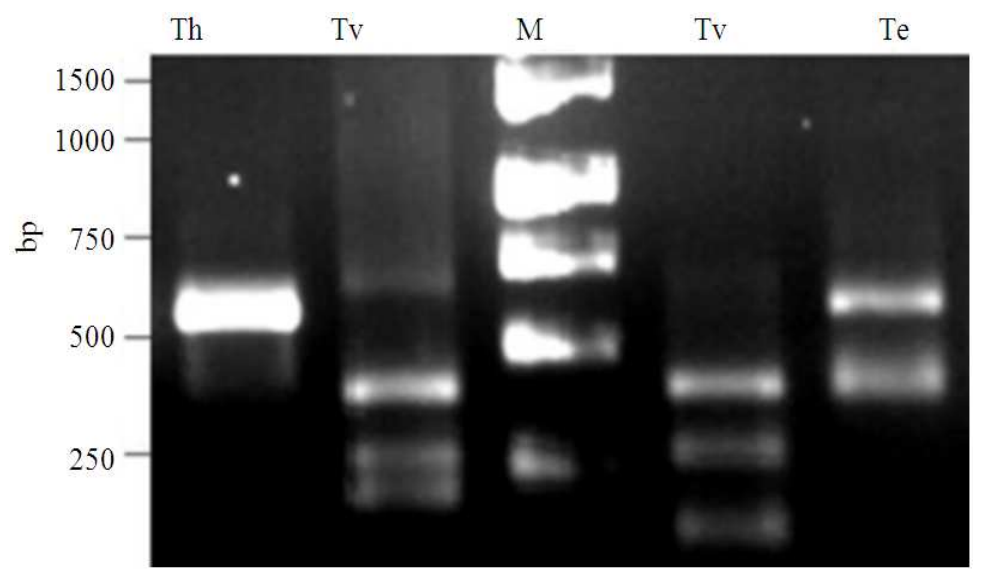

Fig. 2. BglI restriction analysis of the $18 \mathrm{Ssu}$ seminested PCR products for Trypanosome species on $2 \%$ agarose electrophoresis. Th: T. theileri; Tv: T. vivax and Te: T. evansi 
Table 1. Comparison of trypanosome infection frequency by MHCT and PCR-RFLP

\begin{tabular}{llrlr}
\hline & Bovines & & Buffaloes \\
Test & N & $(\%)$ & N & $(\%)$ \\
\hline MHCT $(+)$ & $(6 / 120)$ & 5 & $(5 / 86)$ & 6 \\
PCR $(+)$ & $(54 / 120)$ & 45 & $(39 / 86)$ & 45 \\
T. vivax & $(28 / 120)$ & 23 & $(24 / 86)$ & 28 \\
T. evansi & $(13 / 120)$ & 11 & $(5 / 86)$ & 6 \\
T. theileri & $(13 / 120)$ & 11 & $(10 / 86)$ & 12 \\
\hline
\end{tabular}

Table 2. Clinical features of trypanosome infection by etiological specie in bovines and buffaloes

\begin{tabular}{|c|c|c|c|c|c|c|c|c|c|c|c|}
\hline \multirow[b]{3}{*}{ Parameter } & & \multicolumn{4}{|l|}{ T. vivax } & \multicolumn{2}{|l|}{ T. evansi } & \multicolumn{2}{|l|}{ T. theileri } & \multicolumn{2}{|l|}{ Negatives $^{* *}$} \\
\hline & & \multicolumn{2}{|l|}{ Bovines } & \multicolumn{2}{|l|}{ Buffaloes } & \multirow{2}{*}{$\begin{array}{l}\text { Bovines } \\
\text { PCR }(n=13)\end{array}$} & \multirow{2}{*}{$\begin{array}{l}\text { Buffaloes } \\
-\mathrm{PCR}(\mathrm{n}=5)\end{array}$} & \multirow{2}{*}{$\begin{array}{l}\text { Bovines } \\
\mathrm{PCR}(\mathrm{n}=13)\end{array}$} & \multirow{2}{*}{$\begin{array}{l}\text { Buffaloes } \\
\text { PCR }(n=10)\end{array}$} & \multirow{2}{*}{\begin{tabular}{l} 
Bovines \\
\hdashline$(\mathrm{n}=11)^{* *}$
\end{tabular}} & \multirow{2}{*}{\begin{tabular}{l} 
Buffaloes \\
\hdashline$(\mathrm{n}=15)$
\end{tabular}} \\
\hline & & MHCT $(n=6)$ & PCR $(n=28)$ & MHCT $(\mathrm{n}=5)$ & $\operatorname{PCR}(n=24)$ & & & & & & \\
\hline Variables/RS* & Categories & (n) $\%$ & (n) $\%$ & (n) $\%$ & (n) $\%$ & (n) $\%$ & (n) $\%$ & (n) $\%$ & (n) $\%$ & (n) $\%$ & (n) $\%$ \\
\hline \multirow{4}{*}{ PCV28-36\% } & Normal & (4) 66,7 & (18) 64,3 & (1) 20 & (15) 62,5 & (12) 92,3 & (4) 80 & (11) 84,6 & (9) 90 & (11) 100 & (15) 100 \\
\hline & Mild anemia* & (2) 33,0 & (8) 28,6 & (4) 80 & (6) 25 & (1) 7,7 & (1) 20 & (0) 0 & (0) 0 & (0) 0 & (0) 0 \\
\hline & Moderarte anemia* & (0) 0 & (2) 7,1 & (0) 0 & (3) 12,5 & (0) 0 & (0) 0 & (2) 15,4 & (1) 10 & (0) 0 & (0) 0 \\
\hline & Severe anemia* & (0) 0 & (0) 0 & (0) 0 & (0) 0 & (0) 0 & (0) 0 & (0) 0 & (0) 0 & (0) 0 & (0) 0 \\
\hline WBC & Normal & (1) 16,7 & (21) 75,1 & (3) 60 & (20) 83,33 & (11) 84,6 & (5) 100 & (11) 84,6 & (8) 80 & (11) 100 & (15) 100 \\
\hline \multirow[t]{2}{*}{$5-13.3 \mathrm{E} 10^{3} / \mathrm{mm}^{3}$} & Leucopenia & (4) 66,7 & (5) 17,9 & (2) 40 & (3) 12,5 & (2) 15,4 & (0) 0 & (1) 7,7 & (2) 20 & (0) 0 & (0) 0 \\
\hline & Leukocytosis & (1) 16,7 & (2) 7,1 & (0) 0 & (1) 4,17 & (0) 0 & (0) 0 & (1) 7,7 & (2) 0 & (0) 0 & (0) 0 \\
\hline Lymphocytes & Normal & (1) 16,7 & (21) 75 & (3) 60 & (17) 70,83 & (10) 76,9 & (4) 80 & (12) 92,3 & (7) 70 & (11) 100 & (10) 66.66 \\
\hline \multirow{2}{*}{$45-75 \%$} & Lymphopenia & (4) 66,7 & (4) 14,3 & (2) 40 & (7) 29,17 & (3) 23,1 & (1) 20 & (0) 0 & (3) 30 & (0) 0 & (2) 13.33 \\
\hline & Lymphopenia & (1) 16,7 & (3) 10,7 & (0) 0 & (0) 0 & (0) 0 & (0) 0 & (1) 7,7 & (0) 0 & (0) 0 & (3) 20 \\
\hline Neutrophils & Normal & (5) 83,3 & (20) 71,4 & (4) 80 & (15) 62,5 & (13) 100 & (4) 80 & (8) 61,5 & (9) 90 & (11) 100 & (9) 60 \\
\hline \multirow[t]{2}{*}{$15-45 \%$} & Neutropenia & (0) 0 & (0) 0 & (0) 0 & (0) 0 & (0) 0 & (0) 0 & (0) 0 & (0) 0 & (0) 0 & (6) 40 \\
\hline & Neutrophilia & (1) 16,7 & (8) 28,6 & (1) 20 & (9) 37,5 & (0) 0 & (1) 20 & (5) 38,5 & (1) 10 & (0) 0 & (0) 0 \\
\hline Platelets & Normal & (1) 16,7 & (3) 10,7 & (2) 40 & (7) 29,17 & (2) 15,4 & (0) 0 & (0) 0 & (4) 40 & (6) 54.5 & (9) 60 \\
\hline $252-724 \mathrm{E}$ & Thrombocytopenia & (1) 16,7 & (14) 50 & (3) 60 & (15) 62,5 & (10) 76,9 & (5) 100 & (10) 76,9 & (5) 50 & (3) 27.3 & (1) 6.66 \\
\hline $103 / \mathrm{mm}^{3}$ & Thrombocytosis & (4) 66,7 & (11) 39,3 & (0) 0 & (2) 8,33 & (1) 7,7 & (0) 0 & (3) 23,1 & (1) 10 & (2) 18.2 & (5) 33.33 \\
\hline Lymphadenitis & & (3) 50 & (12) 42,9 & (0) 0 & (0) 0 & (0) 0 & (0) 0 & (2) 15,4 & (0) 0 & (0) 0 & (0) 0 \\
\hline Motor coordination & & (0) 0 & (1) 3,6 & (0) 0 & (1) 4,16 & (0) 0 & (1) 20 & (1) 7,7 & (0) 0 & (0) 0 & (0) 0 \\
\hline Weight loss & & (6) 100 & (25) 89,3 & (2) 40 & (12) 50 & (3) 23,1 & (1) 20 & (17) 53,9 & (2) 20 & (4) 36.4 & (0) 0 \\
\hline Membrane status $=2.5$ & & (3) 50 & (20) 71,4 & (0) 0 & (4) 16,6 & (2) 15,4 & (1) 20 & (8) 61,5 & (0) 0 & (0) 0 & (6) 40 \\
\hline Body condition $=2.5$ & & (6) 100 & (26) 92,9 & (2) 40 & (12) 50 & (7) 53,9 & (5) 100 & (8) 61,5 & (2) 20 & (4) 36.4 & (4) 26.66 \\
\hline
\end{tabular}

*RR: Reference Range. Hematological RR from Jain (1993); **Negative animals for trypanosome sp., Anaplasma marginale and babesia sp. By MHCT and PCR

Table 2 summarizes the most relevant clinical and hematological features of Trypanosomiasis (according to the Trypanosoma species), characterized through bivariate analysis of the infected animals. It is worth pointing out that parasitemia was only detected by MHCT in cases of T. vivax infection, in both species of animals. In cattle, the presence of Trypanosoma $s p$. detected by PCR was associated with female sex ( $\mathrm{P}=$ 0.006; OR 15.4 IC95\% 1.3-189.6), adult cow ( $\mathrm{P}=$ 0.006), low PCV $(\mathrm{P}=0.04)$ and high concentration of animals per hectare $(\mathrm{P}=0.03)$. Infection specifically by T. vivax was significantly associated with mild anemia $(\mathrm{P}$ $=0.002)$, weight loss $(\mathrm{P}=0.02)$, lymphadenitis $(\mathrm{P}=$ $0.04)$ and high concentration of animals per hectare $(\mathrm{P}=$ 0.04). The infection of bovines by $T$. evansi was significantly associated with the concomitant presence of Anaplasma sp. $(\mathrm{P}=0.04)$, low body condition score $(\mathrm{P}=$ $0.03)$, weight loss $(\mathrm{p}<0.001)$, Bos indicus race $(\mathrm{p}<0.001)$ and high concentration of animals per hectare $(\mathrm{p}<0.001)$.

The significance of the associated variables with positive PCR was determined using a logistic regression model multivariate analysis. In this way, infection by Trypanosoma $s p$. in buffaloes was found to be associated with mild anemia (OR 3.4 IC95\%, $\mathrm{P}=0.02)$ and high concentration of animals per hectare (OR $0.3 \mathrm{IC} 95 \%, \mathrm{P}=$ $0.005)$. The variables female sex $(P=0.03)$ and PCV $(P$ $=0.01)$ showed significant differences between buffaloes infected by $T$. vivax and non-infected animals The infection of buffaloes by $T$. evansi was associated with the presence of macroplatelets $(\mathrm{P}=0.02)$ and impaired motor coordination $(\mathrm{P}=0.04)$ while old age $(\mathrm{P}=0.02)$ and thrombocytopenia $(\mathrm{P}=0.02)$ were found to be associated with $T$. theileri infection in buffalo.

\section{DISCUSSION}

Currently, there exists no Gold Standard Method for an accurate species-specific diagnosis of South American Animal Trypanosomiasis from field samples. The very low sensitivity of the parasitological method based on parasite visualization by light microscopy (MHCT, BCT and PBS) is widely recognized. Moreover, for Trypanosomiasis, the diagnostic tools based on amplification of the parasite's DNA have been extensively recognized for their high detection capability (below one parasite per $\mathrm{mL}$ ), therefore their sensitivity is 
close to $100 \%$ (De Souza Pimentel et al., 2012). One of the most widely used methods for molecular characterization of the etiological species involved in Animal Trypanosomiasis is the assay previously described by (Geysen et al., 2003). This tool is based on the double restriction of $18 \mathrm{Ssu}$ semi-nested PCR products, leading to excellent performance in the discrimination of most relevant Trypanosome species involved in African Animal and Human Trypanosomiasis. However, this test presents limitations for the adequate differentiation of T. evansi from T. vivax in the sickness of domestic large ruminants from South America. This restriction is associated in part with the evolutionary differentiation of the rDNA sequences between $T$. vivax forms present in East Africa and those found in America (Rodrigues et al., 2008; Cortez et al., 2009).

Nevertheless, some advances have been made towards overcoming these diagnostic limitations. Mekata et al. (2009) designed a test to detect T. evansi from Bolivian and Peru bovines by PCR using Expression-Site-Associated Genes 6 (ESAG-6) as DNA template. In other work, the identification of $T$. vivax isolated from Pernambuco's cattle samples (Brazil) was performed by a previously described PCR (Geysen et al., 2003) coupled with amplicon sequencing (De Souza Pimentel et al., 2012). For T. vivax diagnosis in South American animals, PCR assays have also been carried out based on cathepsin L-like (CatL-like) cysteine proteases genes or on spliced-leader gene intergenic sequences (Cortez et al., 2009).

In this study, we optimized a new PCR-RFLP for the accurate diagnosis of Animal Trypanosomiasis in South America, with a species-specific resolution. For that purpose, two new primers were constructed based on 18 Ssu rDNA sequences reported for South American isolates of $T$. vivax, T. evansi and T. theileri. These primers were used for a semi-nested PCR assay based on the sequences listed. The products of the above reaction were restricted with $B g l \mathrm{I}$ in a two and half hour reaction with good resolution for the three species on a simple $2 \%$ agarose gel stained with SYBR Gold (Invitrogen Life Technologie) or even with Ethidium Bromide (Promega). For T. vivax, T. evansi and T. theileri, the $B g l \mathrm{I}$ restriction reaction generated profiles characterized by 3,2 and 1 fragments, respectively (Fig. 2). Furthermore, it is worth noting that the described reaction from this study presents better global performance with improved thermodynamic conditions in the PCR and is less expensive (one endonuclease instead of two) and less time consuming (with a $2.5 \mathrm{~h}$ RFLP instead of $14 \mathrm{~h}$ ) than the previously reported PCRRFLP (Geysen et al., 2003).
We validated this new methodology on whole blood samples collected from bovines and water buffaloes naturally infected in the Colombian Magdalena Medio region. The application of the PCR optimized protocol on field samples showed a general infection frequency of $45 \%$ by Trypanosoma sp., from the sampled bovines and buffaloes, while the traditional parasitological methods (BCT, MHCT and PBS) were positive in less than $6 \%$ of the samples. In this way, the PCR described had sensitivity eight times better than microscopy methods.

The detection limit was estimated at $0.1 \mathrm{ng}$ by Nanodrop 2000c software (Thermo Scientific) for T. vivax or T. evansi DNA (equivalent parasitemia of 1 trypanosome $/ \mathrm{mL}$ ). For the specificity of the assay, PCR assays were performed using T. cruzi, Anaplasma sp. and Babesia sp. DNAs as templates with no band amplification.

A $B g l$ I enzyme restriction for the amplicons showed natural infection of bovines and buffalo from the Colombian Magdalena Medio region by three Trypanosome species, of which $T$. vivax was the most common, being responsible for more than half of all the infections. T. evansi was identified in 24 and $12 \%$ of the infected cattle and buffalo, respectively. Infection by the non-pathogenic $T$. theileri was identified in approximately $25 \%$ of the PCR positive animals for Trypanosoma. The sequencing of the $T$. vivax amplicons showed high homology (99\%) with the sequences of the $18 \mathrm{~S}$ genes from the Brazilian and Venezuelan isolates of $T$. vivax (HM209400, AY362546, AY363164 and AY363165).

In the global analysis of the bovine trypanosomiasis we found that Bos indicus was the most susceptible race. Furthermore, bovine females were 15 times more likely to be PCR positive for Trypanosoma $s p$. than males. In accordance with this finding, 71.4, 76.9 and $100 \%$ of the $T$. vivax, T. theileri and T. evansi cattle infected were females, respectively. A similar finding was reported previously for $T$. evansi infection in camels, where this association was related to the additional stress in females due to lactation and successive pregnancies (Singh et al., 2004).

It is worth mentioning that $T$. vivax was identified as the etiological agent in all of the acute infection cases. In bovines, T. vivax acute trypanosomiasis was found to be associated with leukopenia $(<5000 / \mathrm{mm} 3)$, lymphopenia and thrombocytosis. Mild anemia (20-26\% of PCV) was present in a third of the cases. These results are in accordance with previous reports, which noted that the $T$. vivax infection detected by BCT reduced PCV levels in infected cattle (Mbahin et al., 2013). Upon physical examination, the majority of these animals (50-100\%) displayed pale mucous membranes, impaired body 
condition scores $(\leq 2.5)$, increase of capillary filling time $(\geq 2.5 \mathrm{sec})$ and lymphadenitis. A different clinicalhematological profile was evidenced in buffaloes that presented an acute infection, characterized by better mean weight, membrane status and body condition score than those found in cattle. Lymphadenitis did not display a significant association. Similarly, leukopenia and lymphopenia were less frequent in cases of acute trypanosomiasis among the buffaloes however the positive parasitemia was associated with thrombocytopenia and mild anemia in 60 and $80 \%$ of the cases.

Moreover, most of the cases of T.vivax infection $(71 \%)$ were classified as chronic infection (MHCT negative and PCR positive). In terms of clinical presentation, infected cattle presented a range of hematological and clinical signs varying from completely asymptomatic to severe hematologic alterations or death. These findings are in agreement with previous reports (Osório et al., 2008; Batista et al., 2012). Nevertheless, the chronic infection was found to be associated with pale mucous membranes, progressive weight loss, diminished body condition score, lymphadenitis and thrombocytopenia. In a minor percentage of animals, chronic infection displayed severe manifestations with mild to moderate anemia (35.7\%), pancytopenia and emaciation, suggesting the progress of the sickness. On the other hand, buffalo with chronic infection by $T$. vivax presented better general conditions than those described for cattle, with no lymphadenitis and with a minor percentage of animals with weight loss and deterioration of body condition score and membrane status. Thrombocytopenia was also found in cases of chronic infection among buffalo but was accompanied by macroplatelets. These findings indicate that buffalo may have better capability to control the Trypanosome infection than bovines as reported by (Namangala, 2012). Nevertheless, a very small percentage (1/86, $1.2 \%$ ) of buffalo in the chronic phase with moderate anemia, but with apparently acceptable general conditions, suffered a sudden death.

Infection by $T$. evansi displayed an enzootic behavior with asymptomatic presentation in most of the cases and with no acute cases. This infection was significantly associated only with thrombocytopenia and impairment of body condition in the cattle and the buffalo sampled. It is worth mentioning that, in this study, the presence of T. evansi detected by PCR was not found to be significantly associated with impairment of motor coordination since this clinical symptom was only evidenced in one infected buffalo.

In cattle and buffalo the presence of $T$. theileri detected by PCR was associated with normality in all hematological parameters, with the exception of the platelet counts, which were found to be lower in $76.9 \%$ of the cases. In cattle, impairment of body condition was also observed in most $(61 \%)$ of the animals infected by T. theileri. These clinical and hematological features of this infection corroborate the hypothesis that presumes the opportunistic behavior of this parasite in cattle (Lee et al., 2013). However, a specific test able to differentiate this species will avoid unnecessary treatments for this non-pathogenic Trypanosome.

In the present study, the majority of the $T$. evansi $(100 \%)$ and $T$. vivax $(71 \%)$ infected animals were characterized by very low parasitemia only detected by PCR. This result suggests that mechanical transmission by biting flies cannot be the most important mechanism of infection in this region. Dávila et al. (2003) propose that bovines themselves could be acting as reservoirs for T. vivax. Nevertheless, in this study, buffalo presented more subclinical alterations of clinical and hematological parameters than cattle, suggesting a better tolerance of infection and their possible role as reservoir. However, our results indicate that other additional factors such as congenital and iatrogenic transmission could be contributing significantly to the perpetuation of the parasite in the Colombian Magdalena Medio region. This latter finding becomes even more relevant when taking into account that Colombian livestock production is characterized by uncontrolled migration of animals among regions of the country (with no strict measures of hygiene) and the common practice of using shared needles for parenteral proceedings. Further work should be performed in order to clarify the role of these factors in the epidemiological profile of the Trypanosome infection in this area because of their relevance in the formulation of appropriate policies for control of this infection.

\section{CONCLUSION}

In this study, the standardized PCR-RFLP presented high sensitivity and high specificity for $T$. vivax, T. evansi and T. theileri detection. The PCR achieved sensitivity eight times better than microscopy methods. For species identification, restriction analysis with $B g l \mathrm{I}$ differentiated between infection by three different Trypanosome species (56, 25 and $19 \%$ for T. vivax, T. theileri and T. evansi, respectively) with good resolution on field samples from bovines and buffalo. The clinical presentation of Trypanosomiasis by $T$. vivax in the sampled animals ranged from asymptomatic to severe hematological alterations or death, however infected buffalo 
displayed better general conditions than those described for cattle. Acute trypanosomiasis was detected in $11 \%$ of the cases $(6 / 54)$, with $T$. vivax as the only etiological agent. On the other hand, infection by $T$. evansi displayed an enzootic behavior significantly associated with thrombocytopenia and impairment of body condition. Finally, $T$. theileri evidenced an opportunistic behavior and was associated with normality in all the hematological and physical examination parameters studied, with the exception of thrombocytopenia and impairment of body condition.

\section{ACKNOWLEDGEMENT}

This study was supported by grants from the Vicerrectoría de Investigaciones-Universidad de Santander (Project Number 044-11). We would like to thank Prof. María C. Vásquez, Prof. Wilson F. Díaz and the owner and workers of the participant farms for their support during the collection of samples. We are grateful to $\mathrm{Mr}$. Emile Blanchette for assistance in proofreading of this study.

\section{REFERENCES}

Altschul, S.F., W. Gish, W. Miller, E.W. Myers and D.J. Lipman et al., 1990. Basic local alignment search tool. J. Mol. Biol., 215: 403-410. DOI: 10.1006/jmbi.199 0.9999

Batista, J.S., C.M. Rodrigues, R.G. Olinda, T.M. Silva and R.G. Vale et al., 2012. Highly debilitating natural Trypanosoma vivax infections in Brazilian calves: epidemiology, pathology and probable transplacental transmission. Parasitol. Res., 110: 7380. DOI: $10.1007 / \mathrm{s} 00436-011-2452-y$

Cassalett, E.R., V. Julio, J.L. Parra and R.M. Baldrich, 2011. Diagnóstico y caracterización molecular de infecciones naturales por Trypanosoma spp. en bovinos de la Orinoquía Colombiana. Corpoica Cien. Tec. Agrop., 12: 86-91.

Coles, E.H., 1986. Veterinary Clinical Pathology. 4th Edn., WB Saunders Co, Philadelphia, ISBN-10: 0721618286, pp: 486.

Cortez, A.P., A.C. Rodrigues, H.A. Garcia, L. Neves and J.S. Batista et al., 2009. Cathepsin L-like genes of Trypanosoma vivax from Africa and South Americacharacterization, relationships and diagnostic implications. Mol. Cell Probe., 23: 44-51. DOI: 10.1016/j.mcp.2008.11.003
Dávila, A.M.R., H.M. Herrera, T. Schlebinger, S.S. Sou-za and Y.M. Traub-Cseko et al., 2003. Using PCR for unraveling the cryptic epizootiology of livestock trypanosomosis in the Pantanal, Brazil. Vet. Parasitol., 117: 1-13. DOI: 10.1016/j.vetpar.2003.08.002

Desquesnes, M., P. Holzmuller, D.H. Lai, A. Dargantes and Z.R. Lun et al., 2013. Trypanosoma evansi and Surra: a review and perspectives on origin, history, distribution, taxonomy, morphology, hosts and pathogenic effects. BioMed. Res. Inter., 2013: 194176-194197. DOI: 10.1155/2013/194176

De Souza Pimentel, D., C.A. Do Nascimento Ramos, R.A.D.N. Ramos, F.R. de Araújo and M.L. Borba et al., 2012. First report and molecular characterization of Trypanosoma vivax in cattle from state of Pernambuco, Brazil. Vet. Parasitol., 185: 286-289. DOI: 10.1016/j.vetpar.2011.10.019

Delespaux, V., F. Ayral, D. Geysen and S. Geerts, 2003. PCR-RFLP using Ssu-rDNA amplification: Applicability for the diagnosis of mixed infections with different trypanosome species in cattle. Vet. Parasitol., 117: 185-193. DOI: 10.1016/j.vetpar.2003.08.004

Geysen, D., V. Delespaux and S. Geerts, 2003. PCRRFLP Using Ssu-rDNA amplification as an easy method for species-specific diagnosis of Trypanosoma species in cattle. Vet. Parasitol., 110: 171-180. DOI: 10.1016/S0304-4017(02)00313-8

Holland, W.G., F. Claes, L.N. My, N.G. Thanh and P.T. Tam et al., 2001. A comparative evaluation of parasitological tests and a PCR for Trypanosoma evansi diagnosis in experimentally infected water buffaloes. Vet. Parasitol., 97: 23-33. DOI: 10.1016/.0304-4017(01)00381-8

Jain, N.C., 1993. Essentials of Veterinary Hematology. 1stw Edn., Wiley, Philadelphia, ISBN-10: 081211437X, pp: 417.

Lee, Y.F., C.C. Cheng, J.S. Chen, N.N. Lin and Y.W. Hung et al., 2013. Evidence of intracellular stages in Trypanosoma Megatrypanum theileri in nonphagocytic mammalian cells. Vet. Parasitol., 191: 228-239. DOI:10.1016/j.vetpar.2012.08.027

Mbahin, N., H. Affognon, J. Andoke, M. Tiberius and D. Mbuvi et al., 2013. Parasitological prevalence of bovine trypanosomosis in Kubo division of Kwale county of coastal: Baseline survey. Am. J. Anim. Vet. Sci., 8: 28-36. DOI: 10.3844/ajavsp.2013.28.36

Mekata, H., S. Konnai, W.H. Witola, N. Inoue and M. Onuma et al., 2009. Molecular detection of trypanosomes in cattle in South America and genetic diversity of Trypanosoma evansi based on expressionsite-associated gene 6. Infect. Genet. Evol., 9: 13011305. DOI: 10.1016/j.meegid.2009.07.009

AJAVS 
Murray, M., P.K. Murray and W.I.M. McIntyre, 1977. An improved parasitological technique for the diagnosis of African Trypanosomiasis. T. Roy. Soc. Trop. Med. H., 71: 325-328. DOI: 10.1016/00359203(77)90110-9

Namangala, B., 2012. Contribution of innate immune responses towards resistance to African trypanosome infections. Scand. J. Immunol., 75: 5-15. DOI: 10.1111/j.1365-3083.2011.02619.x

Osório A.L.A.R., C.R. Madruga, M. Desquesnes, C.O. Soares and L.R.R. Ribeiro et al., 2008. Trypanosoma (Duttonella) vivax: its biology, epidemiology, pathogenesis and introduction in the new world-a review. Mem. I. Oswaldo Cruz, 103: 1-13. DOI: $10.1590 /$ S007402762008000100001

Otte, M.J., J.Y. Abuabara and E.A. Wells, 1994. Trypanosoma vivax in colombia: Epidemiology and production losses. Trop. Anim. Health Pro., 26: 146156. DOI: $10.1007 / \mathrm{BF} 02241071$
Rodrigues, A.C., L. Neves, H.A. Garcia, L.B. Viola and A. Marcili et al., 2008. Phylogenetic analysis of Trypanosoma vivax supports the separation of South American/West African from East African isolates and a new $T$. vivax-like genotype infecting a nyala antelope from Mozambique. Parasitology, 135: 1317-1328. DOI: 10.1017/S0031182008004848

Singh, N., K.L.M. Pathak and R. Kumar. 2004. A comparative evaluation of parasitological, serological and DNA amplification methods for diagnosis of natural Trypanosoma evansi infection in camels. Vet. Parasitol., 126: 365-373. DOI: 10.1016/j.vetpar.2004.08.013

Wells, E.A., L.E. Ramírez and A. Betancourt, 1982. Trypanosoma vivax in Colombia: Interpretation of field results. Trop. Anim. Health Prod., 14: 141-150. DOI: $10.1007 / \mathrm{BF} 02242144$

Woo, P.T.K., 1970. The haematocrit centrifuge technique for the diagnosis of African trypanosomiasis. Acta Trop., 27: 384-386. 